\title{
Pre-term birth in women exposed to Cushing's disease: the baby-cush study
}

\author{
Clarisse Hochman', Justine Cristante ${ }^{2}$, Aurore Geslot' ${ }^{3}$, Sylvie Salenave ${ }^{4}$, Emmanuel Sonnet ${ }^{5}$, Claire Briet ${ }^{6}$, \\ Anne Bachelot ${ }^{7}$, Nicolas Chevalier ${ }^{8}$, Olivier Gilly ${ }^{9}$, Thierry Brue ${ }^{10,11}$, Samy Hadjadj', Veronique Kerlan5, \\ Philippe Chanson ${ }^{4}$, Delphine Vezzosi ${ }^{3}$, Olivier Chabre², Delphine Drui(i) ${ }^{1}$ and Frederic Castinetti ${ }^{10,11}$
}

'Service d'Endocrinologie, L'institut du thorax, CHU de Nantes, Nantes, Pays de la Loire, France, ${ }^{2}$ Endocrinologie, Diabétologie et Maladies Métaboliques, CHU de Grenoble, Grenoble, France, ${ }^{3}$ Service d'Endocrinologie, Hôpital Larrey, Toulouse, France, ${ }^{4}$ Assistance Publique-Hôpitaux de Paris, Hôpital Bicêtre, Service d'Endocrinologie et des Maladies de la Reproduction, Centre de Référence des Maladies Rares de l'Hypophyse and Université Paris-Saclay, Univ. Paris-Sud, Inserm, Signalisation Hormonale, Physiopathologie Endocrinienne et Métabolique, Le Kremlin-Bicêtre, Paris, France, ${ }^{5}$ Service d'Endocrinologie-Diabète et Maladies Métaboliques, Hôpital de la Cavale Blanche, CHU de Brest, Brest, France, ${ }^{6}$ Service Endocrinologie, Diabétologie et Nutrition, Institut Mitovasc, CHU d'Angers, Angers, France, ${ }^{7}$ Service d'Endocrinologie et Médecine de la Reproduction, IE3M, Hôpital Universitaire Pitié Salpêtrière, Sorbonne Université, Paris, France, ${ }^{8}$ Service d'Endocrinologie, CHU de Nice, Nice, France, ${ }^{9}$ Service des Maladies Métaboliques et Endocriniennes, CHU Nîmes, Nimes, France, ${ }^{10} \mathrm{Aix}$-Marseille Université, Institut National de la Santé et de la Recherche Médicale (INSERM), U1251, Marseille Medical Genetics (MMG), Institut Marseille Maladies Rares (MarMaRa), Marseille, France, and ${ }^{11}$ Assistance Publique-Hôpitaux de Marseille (AP-HM), Department of Endocrinology, Hôpital de la Conception, Centre de Référence des Maladies Rares de I'hypophyse HYPO, Marseille, France

Correspondence should be addressed to F Castinetti Email frederic.castinetti@ap-hm.fr

\begin{abstract}
Design: Hypercortisolism during pregnancy is a risk factor for prematurity. Long-term exposure to hypercortisolism may lead to permanent comorbidities, such as hypertension or diabetes, even after remission. Our aim was to determine whether women with a history of Cushing's disease (and being eu-, hypo- or hypercortisolic at the time of pregnancy) had the same risks of comorbidities, and especially prematurity, during pregnancy.

Methods: It was a retrospective multicentric study focusing on mothers with a history of Cushing's disease or diagnosed during pregnancy, followed in French tertiary referral centers. We compared the outcomes of pregnancies depending on the cortisolic status at the time of pregnancy.

Results: A total of 60 patients (78 pregnancies including 21 with hypercortisolism, 32 with hypocortisolism and 25 in eucortisolism in 25 ) were evaluated. The overall rate of preterm birth was $24.3 \%$, with a peak in women diagnosed during pregnancy (62.5\%), a high risk in hypercortisolic (33\%) and hypocortisolic (19.3\%), and a low risk (8\%) in eucortisolic women Gestational diabetes and hypertension were observed in $21 \%$ and $10.4 \%$ of the whole cohort, with a higher risk in hypercortisolic women. Cesarean delivery was performed in $33.7 \%$ of the cohort.

Conclusions: Being non-eucortisolic at the time of pregnancy increases the risk of prematurity and comorbidities compared to the general population. Women with a history of Cushing's disease should thus be carefully monitored during pregnancy. The high rate of cesarean delivery emphasizes the fact that these pregnancies should always be considered at risk.
\end{abstract}

European Journal of Endocrinology

(2021) 184, 469-476
Published by Bioscientifica Ltd. 


\section{Introduction}

While pregnancy is a rare event in patients with Cushing's syndrome (CS), it is even less frequent in patients with Cushing's disease (CD). Indeed, only $15-40 \%$ of the cases of hypercortisolism during pregnancy are due to CD (the remaining being mainly adrenal adenomas), while it represents $70-80 \%$ of the etiologies of hypercortisolism in other circumstances $(1,2,3)$. The low rate of pregnancy in $\mathrm{CD}$ is probably due to the frequent associated secretion of androgens and also to the fact that the treatment of CD might lead to hypogonadotrophic hypogonadism. This low prevalence likely explains why the detailed outcomes of the pregnancies in women with a history of CD have mainly been analyzed in series of case reports of CS $(4,5$, $6,7,8,9,10,11,12,13)$.

Prematurity has been associated with sociodemographic, obstetric and behavioral characteristics in the general population. Interestingly, it has also been associated with stress, anxiety and depression, features that are frequently seen in patients with CS (14). Moreover, some discordant studies suggested that women with physiologically higher cortisol levels might be at higher risk of prematurity $(15,16)$. As such, one could consider that hypercortisolic women at the time of pregnancy would be at higher risk than eucortisolic and hypocortisolic women to present with pre-term birth. To our knowledge, this point has never been specifically studied via a comparison between women exposed to hypercortisolism and presenting with different cortisolic status at the time of pregnancy due to a previous exposure to hypercortisolism. The most well-documented literature review about women with a history of CS and pregnancy reported the outcomes of 122 patients, including 40 with CD: prematurity (43\%) and intra-uterine growth restriction (21\%) were the most frequent fetal comorbidities. Gestational diabetes, hypertension and prematurity were more frequently observed in hypercortisolic vs eucortisolic women. Of note, rates of complications seemed even higher in patients diagnosed with CS during pregnancy (4). Finally, in a dedicated multicentric study about the outcomes of pregnant patients with adrenal insufficiency, Bothou et al. reported $21.4 \%$ pre-term birth in their cohort (17). While these data brought important clues to optimize the management of CS or adrenal insufficiency during pregnancy, a proper comparison was difficult because these patients were followed in different centers, with different ways of management. Moreover, a comparison with the data of the cohort with each specific country could not be performed.
To try to improve our knowledge of CD and pregnancy, the Baby-Cush study was launched in France as a multicentric retrospective study. Our aim was to analyze the outcome of the pregnancies of patients followed for a history of CD in France. We specifically wanted to determine the risk of preterm birth depending on cortisol status (hypocortisolic, eucortisolic or hypercortisolic) during pregnancy, comparing it to the rates observed in the French population, and also to better characterize the maternal and fetal events occurring during pregnancy. Our hypothesis was that women exposed to previous hypercortisolism, whatever their status at the time of pregnancy, might be at higher risk of complicated pregnancy than the general population. $\mathrm{CD}$ is indeed known to induce sequelae, and we thought that, added to hypertension or diabetes, the risk of preterm birth might be higher in these women because of their history of hypercortisolism.

\section{Patients and methods}

\section{Patients}

The Baby-Cush study was designed as a retrospective multicentric study involving 10 French expert tertiary referral Centers (Angers, Brest, Grenoble, Kremlin-Bicetre, Marseille, Nice, Nimes, Nantes, Paris-La Pitié Salpetriere and Toulouse). Women were included if they had at least one pregnancy and a diagnosis of $\mathrm{CD}$ before or at the time of pregnancy, between January 1989 and January 2020. All patients gave their consent for the use of anonymized data extracted from medical datafiles, as approved by the Ethics Committee of Aix-Marseille University (reference 2020-01-23-02).

\section{Clinical, hormonal and pregnancy outcomes}

The following data were retrieved from datafiles:

- Medical and obstetrical history before pregnancy including diabetes, high blood pressure, miscarriage and previous pregnancies.

- Secretory cortisolic status at the time of pregnancy including data about the management of $\mathrm{CD}$ and ongoing treatments during the pregnancy. Each dataset was fulfilled by the investigator in charge of the patient at the time of inclusion, and he/she defined the cortisolic status at the time of pregnancy based on clinical and hormonal parameters (hypercortisolism was defined by the mean of two to three 24-h urinary 
free cortisol measurements and when necessary, midnight salivary cortisol and/or midnight plasma cortisol; hypocortisolism was defined by 08:00 h plasma cortisol). For hypocortisolic patients, the dose of hydrocortisone at pregnancy onset and the dose changes performed during the pregnancy were also registered.

- Follow-up data of any pregnancy occurring during CD, or after the diagnosis of CD (whatever the treatment), based on maternal (gestational diabetes, high blood pressure, preeclampsia) and fetal (preterm birth, birth weight, APGAR score at 1 and $5 \mathrm{~min}$ ) outcomes. The delivery mode was also noted, as well as the reason in case of cesarean. The diagnosis of high blood pressure and gestational diabetes was based on the guidelines used at the time of the pregnancy: as such, we considered such events when they were noted in the medical records of each patient.

Our primary criterion was the rate of preterm birth (i.e. birth before 37 weeks of gestation) in the whole population. Our secondary criteria were the rate of preterm birth before 34 weeks of gestation, the mode of delivery, the APGAR scores at 1 and $5 \mathrm{~min}$ as well as the rate of high blood pressure, preeclampsia and gestational diabetes. All these data were evaluated for the whole cohort and for the patients depending on their cortisolic status at the time of pregnancy (eucortisolism, hypocortisolism, hypercortisolism). Of note, as some patients were presented with several pregnancies, their secretory status might have changed from one pregnancy to the other; they were thus included several times in different groups.

\section{Statistical analysis}

Quantitative data are reported as mean \pm S.D.. The secretory status (hypercortisolic, eucortisolic or hypocortisolic) at the time of pregnancy and its impact on the outcomes was analyzed with Fisher's exact test, showing the odds ratio (OR) and the 95\% CI. For quantitative data, comparison was performed with one-way ANOVA. All statistical tests were two-tailed, and $P<0.05$ was considered significant. Statistical analyses were performed with R (v3.5.2).

\section{Results}

\section{General characteristics of the population}

A total of 60 patients (78 pregnancies) were included in this retrospective study. CD had been diagnosed at a mean of $5.09 \pm 5.07$ years before pregnancy. Two of our patients changed their cortisol secretory status during the pregnancy because they were operated during the second trimester, we included them in the hypercortisolic group. Eight patients had two pregnancies with different cortisol secretory status: four were in hypo- and then eucortisolism, two in hyper- and then eucortisolism, one in hyper- and then hypocortisolism, and one in eucortisolism and then hypercortisolism. One patient had three pregnancies, the first being hypercortisolic, while the two others being hypocortisolic. Miscarriage was reported in one patient. A total of 76 live births ( 1 neonatal death) was thus reported.

During pregnancy, hypertension was reported in eight patients $(10.4 \%)$, while twice as many patients ( $n=16,21 \%)$ presented with diabetes, and majority of them $(75 \%)$ were treated with insulin (the remaining patients had dietary advice). One third of deliveries ( $n=26,33.7 \%$ ) were done by cesarean: fifteen of these were preterm, including four before 34 weeks of gestation. Cesarean delivery was performed because of disturbed labor progress $(n=7)$, preeclampsia $(n=5)$, fetal bradycardia $(\mathrm{n}=3)$, abnormal fetal position $(n=2)$, cholestasis $(n=1)$ and fetal suffering due to cardiac septal hypertrophy $(n=1)$. Two cesarean deliveries had been planned (missing data for five pregnancies). A higher rate of cesarean delivery (58\%) was reported for hypocortisolic vs eucortisolic patients $(12 \%$ cesarean, $P=0.002)$, while there was a tendency, though not significant, for higher rate in hypercortisolic vs hypocortisolic patients $(23.8 \%$ vs $58 \%, P=0.079)$.

Mean Apgar scores were $9.53 \pm 0.87$ at $1 \mathrm{~min}$ and $9.84 \pm 0.375$ at $5 \mathrm{~min}$. Mean birth weight was $3212 \pm 751.4$ $\mathrm{g}$ (Individual data are shown in Fig. 1).

\section{Outcomes of pregnancies according to cortisolic status}

We compared the outcomes of pregnancies depending on the cortisolic status at the time of pregnancy. A total of 21 pregnancies (27\%) occurred in hypercortisolism, 32 (41\%) in hypocortisolism and 25 (32\%) in eucortisolism.

In the group of hypercortisolic patients, 6 were diagnosed during pregnancy (Supplementary Table 1, see section on supplementary materials given at the end of this article) and 2 shortly after delivery (in the first 3 months). Two patients were operated during the second trimester of pregnancy, while 7 were operated 2-8 months after delivery. In the group of 13 women with a diagnosis of CD before pregnancy, only 3 were on medical treatment (ketoconazole, $n=2$; cabergoline, $n=1$ ) 
A Birth weight (grams)

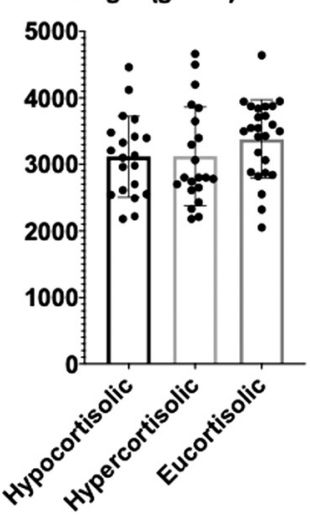

B Pregnancy term (weeks of gestation)

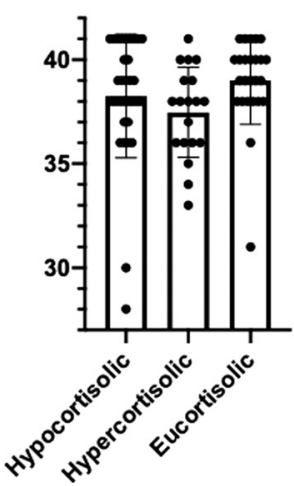

\section{Figure 1}

Individual birth term and pregnancy weight based on cortisolic status. (A) Individual birth weight (median \pm S.D.) in women being hypercortisolic, hypocortisolic or eucortisolic at the time of pregnancy. (B) Individual pregnancy term (median \pm S.D.) in women being hypercortisolic, hypocortisolic or eucortisolic at the time of pregnancy. Note that $\mathrm{C}$ sections were performed in 18 pregnancies of hypocortisolic women (28-41 weeks of gestation, median 38.5 weeks, including 6 preterm), 5 pregnancies of hypercortisolic women (34-38 weeks of gestation, median 37 weeks, including 3 preterm) and 3 pregnancies of eucortisolic women (38, 39 and 40 weeks of gestation).

which was stopped before pregnancy (as pregnancy was planned). All the others $(n=10)$ had Cushing's disease with unequivocal biology in favor of the diagnosis; however, as they presented only moderate consequences of hypercortisolism, none was provided a medical treatment before and/or during pregnancy.

In the group of hypocortisolic patients, 20 (63\%) presented with secondary adrenal insufficiency, while $12(37 \%)$ presented with primary adrenal insufficiency due to bilateral adrenalectomy (details are provided in Table 1). The mean dose of hydrocortisone was $22.0 \pm 7.67 \mathrm{mg}$ vs $23.9 \pm 6.99 \mathrm{mg}$ at the end of pregnancy $(P=0.412)$. Two patients (out of 28 with available data, i.e. $7.1 \%)$ presented an episode of adrenal crisis during the pregnancy: one also had gestational diabetes and delivery occurred at 37 weeks of gestation; the other had no other comorbidity during pregnancy and delivery occurred at 39 weeks of gestation. In these two patients, the dose had been increased during pregnancy, from 30 to 40 , and 20 to $30 \mathrm{mg}$ /day. In the whole group of patients with hypocortisolism, 11 women increased the dose during pregnancy (from 30 to 100\% increase, mean $50 \%)$. The remaining 21 patients had an unchanged dose of hydrocortisone. Finally, one patient presented an adrenal crisis immediately after delivery.

As shown in Table 2, the rate of preterm birth was 33\% in hyper, $19.3 \%$ in hypo- and $8 \%$ in eucortisolic patients. Though the difference in terms of prematurity was not significant between the three groups, there was a tendency for a higher risk of preterm birth in hypercortisolic vs eucortisolic patients $(P=0.059)$. Of note, in the hypercortisolic patients at the time of pregnancy, preterm birth was observed in $5 / 8$ patients $(62.5 \%)$ who were newly diagnosed during or immediately after pregnancy and in $2 / 13$ patients (15.4\%) with a history of CD. For these two patients, both presented hypertension (with preeclampsia in one), and one had a pregnancy denial (diagnosis at 6 months gestation). In the hypocortisolic patients, the mean dose of hydrocortisone at the beginning of pregnancy was not significantly different between preterm vs non-preterm groups $(24 \pm 5.47$ vs $21.1 \pm 7.52 \mathrm{mg} /$ day, $P=0.429$ ). The dose at the end of pregnancy was also not statistically different $(25 \pm 6.1$ vs $24.4 \pm 9.4 \mathrm{mg} /$ day, $P=0.869$ ).

When considering comorbidities of pregnancy, there was a significant increased risk of gestational diabetes in patients with hypercortisolism vs the others $(P=0.008$, $\mathrm{OR}=6.98$; IC $95 \%(1.40 ; 48.13)$ for hyper- vs eucortisolic; $P=0.002, \mathrm{OR}=8.86$; IC $95 \%(1.81 ; 60.42)$ for hyper- vs hypocortisolic). No significant difference was observed for high blood pressure or preeclampsia, although preeclampsia was reported in roughly $10 \%$ patients with hyper- or hypocortisolism, while no such event was reported in eucortisolic patients $(P=0.256)$.

Considering birth, Apgar scores at 1 and $5 \mathrm{~min}$ as well as birth weight were not statistically different among the three groups (Table 2). Following delivery, one newborn presented with a cervical angioma requiring respiratory assistance, one preterm with a ventricular brain hemorrhage; they were both from hypercortisolic mothers and had no sequelae. Two newborns from hypocortisolic mothers presented with abnormal esophagus motility. No specific fetal complication was reported in babies from eucortisolic women. In the three women treated with steroidogenesis inhibitors before pregnancy, no fetal complication was reported.

\section{Discussion}

In women with a history of $\mathrm{CD}$, being non-eucortisolic at the time of pregnancy increases the risk of preterm birth. Indeed, our overall rate of $24.3 \%$ preterm birth 
Table 1 Main characteristics between patients with hypocortisolism due to primary or secondary adrenal insufficiency (SAI).

\begin{tabular}{l}
\hline \\
\hline Patients, $n$ \\
Hydrocortisone dose, mg/day \\
$\quad$ Initial \\
$\quad$ Final \\
Patients with adrenal crises during pregnancy \\
Hypertension \\
Preeclampsia \\
Gestational diabetes \\
C-sections \\
Preterm birth \\
$\quad<37$ weeks \\
$<34$ weeks \\
Birth weight, g \\
Apgar score \\
1 min \\
5 min
\end{tabular}

\begin{tabular}{c}
\hline SAI* \\
\hline 12 \\
$26.67 \pm 7.48$ \\
$29.58 \pm 8.64$ \\
2 \\
1 \\
1 \\
3 \\
6 \\
3 \\
3 \\
0 \\
$2886.67 \pm 468.07$ \\
$9.44 \pm 1.01$ \\
10
\end{tabular}

\begin{tabular}{c}
\hline ACTH deficiency \\
\hline 20 \\
$18.75 \pm 5.09$ \\
$20.83 \pm 8.95$ \\
0 \\
2 \\
2 \\
0 \\
10 \\
5 \\
3 \\
2 \\
$3042.38 \pm 836.86$ \\
$9.38 \pm 1.09$ \\
$9.89 \pm 0.47$
\end{tabular}

\begin{tabular}{c}
\hline P-values \\
\hline- \\
0.001 \\
0.012 \\
0.133 \\
0.999 \\
0.999 \\
0.044 \\
0.758 \\
0.876 \\
- \\
- \\
0.558 \\
0.899 \\
0.490 \\
\hline
\end{tabular}

*Adrenal insufficiency following bilateral adrenalectomy.

was much higher than the rate of $7.5 \%$ reported in the French population and the rate of $8.4 \%$ reported in 57 629 French patients with gestational diabetes $(18,19)$. However, this increased risk was only observed in patients with hypercortisolism at the time of pregnancy (33\% preterm birth), and, to a lesser degree, for patients with hypocortisolism $(19.3 \%$ despite a seemingly adequate replacement therapy by hydrocortisone, varying from 22 to $24 \mathrm{mg}$ /day between the beginning and the end of pregnancy). In contrast, when patients were eucortisolic, the rate of preterm birth was comparable to the one of the French population despite their history of CD (19). In $\mathrm{CD}$, surgery is usually followed by a transient period of corticotroph deficiency: when possible, physicians should thus advice their patients to wait for recovery before pregnancy (a situation which should happen in the majority of cases in the following 1-3 years). Tang et al. recently reported a rate close to our results (26-28\% of preterm birth) in 27 patients with pregnancy associated CD (i.e. CD onset during gestation or within 12 months after delivery or abortion) (5). Caimari et al. reported an even higher rate of pre-term birth (65.8\%), in a review which included $80 \%$ of patients with active hypercortisolism at the time of pregnancy $(2 / 3$ of the cases) (4). Our rate was similar when focusing exclusively on patients newly diagnosed during pregnancy, while the rate of preterm birth was much lower (and comparable to hypocortisolic patients) when only focusing on hypercortisolic patients with a history of CD. Despite that high rate of preterm birth, the birth weight was not statistically different to the French population; this is probably due to a high rate of gestational diabetes in hypercortisolic patients, leading to a relative macrosomia as shown in Fig. 1.

A high rate of metabolic comorbidities was also observed in our cohort. A comparison with the French population (based on 13384 pregnancies in 2016) indeed showed a higher rate of high blood pressure $(10.4 \%$ vs $2.3 \%)$, preeclampsia (6.6\% vs $2 \%$ ) and gestational diabetes (21\% vs $10.8 \%$ ) in our patients (19). Bronstein et al. reported that uncontrolled hypercortisolism during pregnancy was associated with hypertension (68\%), diabetes or glucose intolerance (25\%), preeclampsia (14\%), osteoporosis and fractures (5\%), cardiac failure (3\%), psychiatric disorders (4\%), wound infections $(2 \%)$, and maternal death (2\%) (1). In another review, miscarriage and neonatal or maternal death were also significantly higher compared to our data ( $28 \%$ vs $1.3 \%, 4.9 \%$ vs $1.3 \%$ and $0.8 \%$ vs $0 \%$, respectively) (4). The profiles of patients were however different as these reviews were not specifically focusing on CD but included patients with adrenal carcinoma, ectopic ACTH secretion or adrenal aberrant receptors, groups in which the authors identified a higher rate of mortality and comorbidities than in CD. Moreover, different outcomes in the previous reviews might have been influenced by the older data (19531973), the period of time during which the management and the monitoring of pregnancy were likely different than in the last 30 years. Finally, 17 women presented at least 1 miscarriage before the diagnosis of $\mathrm{CD}$, including 8 women who had 2 or 3 miscarriages (data not shown). This means that a total of 29 miscarriages were observed in our cohort. However, as our study was retrospective, we had no data on the delayed diagnosis. As such, it was 


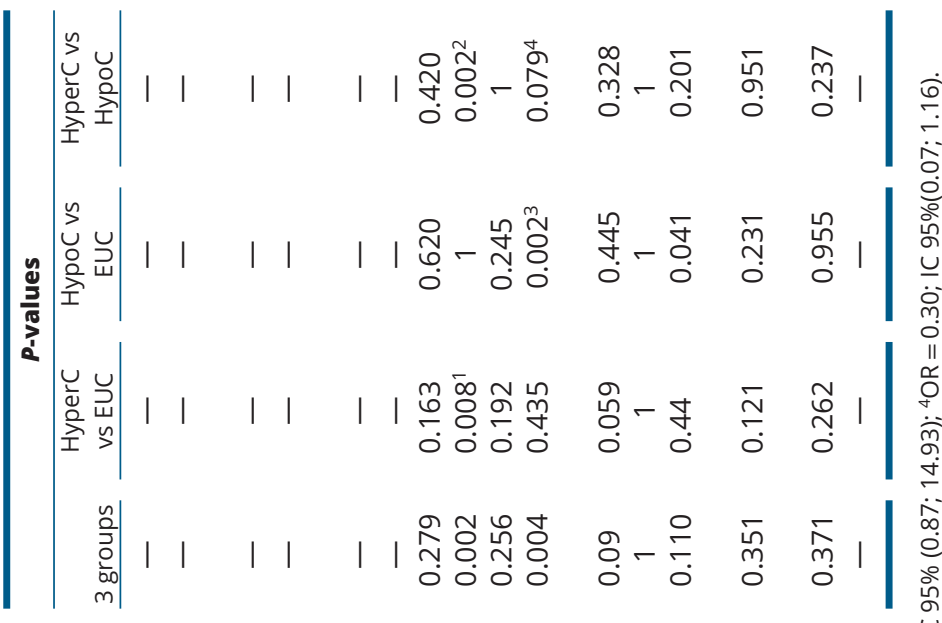

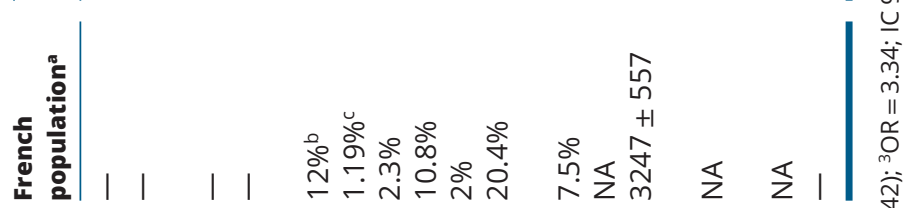

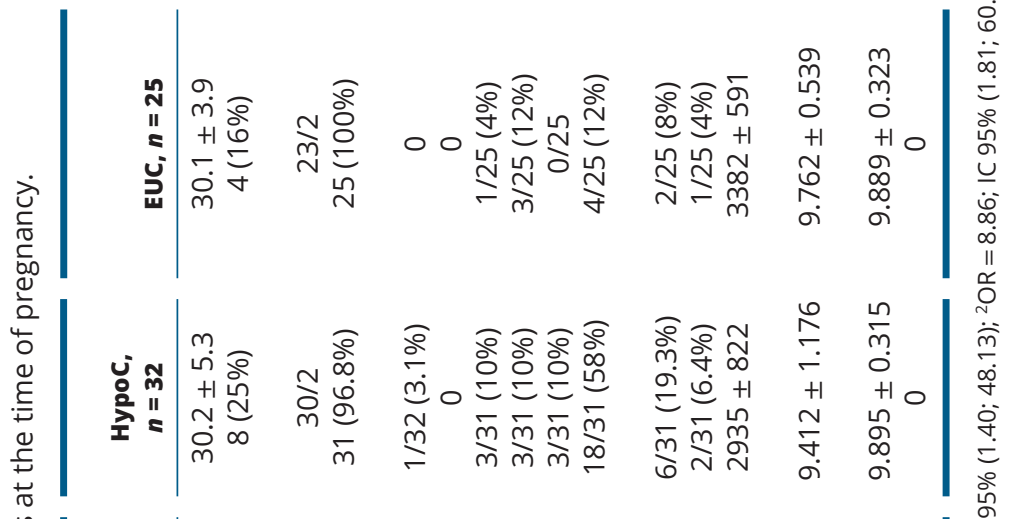

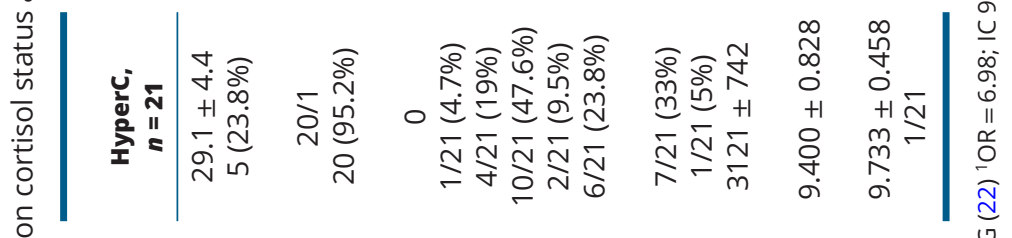

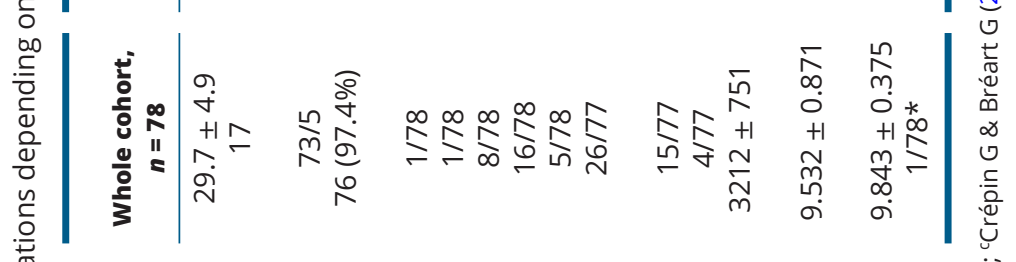

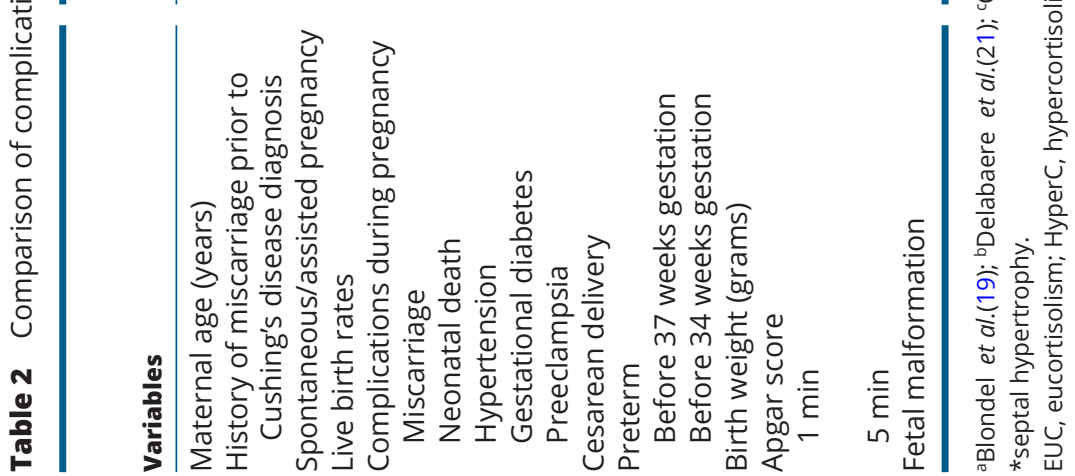


not possible to determine whether these miscarriages happened while being eu- or hypercortisolic (though not yet diagnosed).

When looking at comorbidities in the subgroups based on different secretory status at the time of pregnancy, a significant statistical difference was only observed for gestational diabetes, with a higher risk in hypercortisolic patients compared to the other groups. The rates of high blood pressure and preeclampsia seemed higher in hyper and hypocortisolic patients compared to eucortisolic patients; however, statistical significance could not be shown probably because of an insufficient number of events. Interestingly, the higher rate of cesarean delivery was observed in hypocortisolic patients (53\%). This rate is higher than the one reported in the French population (20\%) (19) but is similar to the one reported by Bothou et al. in a large series of 128 patients with adrenal insufficiency (58\%) (17). The details of the reasons for these cesarean deliveries clearly showed that they had not been performed forthe fear of the obstetricians related to the adrenal insufficiency or the pituitary tumor.

Apart from prematurity, we did not observe any major fetal complication in the three groups of patients. Apgar scores were not statistically different among hyper-, hypo and eucortisolic patients. Increased fetal morbidity had been emphasized by several studies, including intrauterine growth restriction in 20\% cases, stillbirth in $6 \%$ or intrauterine death in $5 \%(1,4)$. These rates were not reported in our cohort. However, all the patients were followed in tertiary referral centers. This was also the case in the study by Bothou et al., in which the authors had not reported any major fetal comorbidity (17). This illustrates the fact that such fragile patients should be closely followed by expert endocrinologists and gynecologists during pregnancy to minimize the risk of comorbidity (other than preterm birth).

To our knowledge, our study is the first to compare three different cortisolic status in terms of pregnancy outcomes. This allowed us to draw some conclusions on the risks of each status but led to relatively small numbers of patients in each group. This likely explains why statistical significance could not be obtained for some parameters such as blood pressure. The retrospective nature of the study led to missing hormonal data for a number of patients. We thus could not determine the levels of exposure in each patient, especially the ones being hypercortisolic at the time of pregnancy. Though we know that all these patients were followed on a regular basis with free cortisol (salivary, and urinary when available), not all descriptive data could be retrieved. However, as these patients were all followed in tertiary referral centers, the monitoring was very similar. This is why we considered that the comorbidities depicted here were due to the disease rather than erroneous or incomplete medical workup during the pregnancy. As this was not the aim of the study, we did not collect data on thyroid status, while T4 levels out of target recommended for central hypothyroidism might have modified the outcomes of pregnancies. Finally, retrospective collection of data did not allow to search for known factors of prematurity such as lower level of education, low income, smoking or genitourinary infections (14).

To conclude, CD markedly modified the outcome of pregnancy, especially when patients were not eucortisolic. Despite the fact that hypercortisolism was probably mild in most of our patients, we reported a high rate of preterm birth and comorbidities, which should make physicians emphasize the need for a proper contraceptive method for these patients. Despite the fact that hypocortisolism was replaced with so-called physiological doses (20), we reported a rate of comorbidities closer to hypercortisolic than eucortisolic patients. This should make us rethink of the appropriate dose of hydrocortisone during pregnancy and probably discuss the need for lower doses of hydrocortisone during pregnancy (even though we did not find any significant difference in terms of dose of hydrocortisone between patients with or without preterm birth). Finally, as eucortisolic patients had the same outcome as the general population, a history of CD should not be considered a risk factor per se for comorbidities during pregnancy, provided eucortisolism is obtained. Perspective should now be focusing on the outcomes of children exposed to mild endogenous or exogenous hypercortisolism during pregnancy to determine potential outcomes for their development.

\section{Supplementary materials}

This is linked to the online version of the paper at https://doi.org/10.1530/ EJE-20-1224.

\section{Declaration of interest}

The authors declare that there is no conflict of interest that could be perceived as prejudicing the impartiality of this study.

\section{Funding}

This research did not receive any specific grant from any funding agency in the public, commercial or not-for-profit sector.

Author contribution statement

D Drui and F Castinetti contributed equally to this work. 


\section{Acknowledgements}

The authors would like to thank the patients who agreed to take part to this study, all the members of the medical team, and lan Darby for English editing.

\section{References}

1 Bronstein MD, Machado MC \& Fragoso MCBV. MANAGEMENT OF ENDOCRINE DISEASE: Management of pregnant patients with Cushing's syndrome. European Journal of Endocrinology 2015173 R85-R91. (https://doi.org/10.1530/EJE-14-1130)

2 Brue T, Amodru V \& Castinetti F. MANAGEMENT OF ENDOCRINE DISEASE: Management of Cushing's syndrome during pregnancy: solved and unsolved questions. European Journal of Endocrinology 2018 178 R259-R266. (https://doi.org/10.1530/EJE-17-1058)

3 Lindsay JR \& Nieman LK. The hypothalamic-pituitary-adrenal axis in pregnancy: challenges in disease detection and treatment. Endocrine Reviews 200526 775-799. (https://doi.org/10.1210/er.2004-0025)

4 Caimari F, Valassi E, Garbayo P, Steffensen C, Santos A, Corcoy R $\&$ Webb SM. Cushing's syndrome and pregnancy outcomes: a systematic review of published cases. Endocrine 201755 555-563. (https://doi.org/10.1007/s12020-016-1117-0)

5 Tang K, Lu L, Feng M, Zhang H, Chen K, Sun X, Zhu H, Wang R \& $\mathrm{Lu} \mathrm{Z}$. The incidence of pregnancy-associated Cushing's disease and its relation to pregnancy: a retrospective study. Frontiers in Endocrinology 202011 305. (https://doi.org/10.3389/fendo.2020.00305)

6 Abbassy M, Kshettry VR, Hamrahian AH, Johnston PC, Dobri GA, Avitsian R, Woodard TD \& Recinos PF. Surgical management of recurrent Cushing's disease in pregnancy: a case report. Surgical Neurology International 20156 S640-S645. (https://doi. org/10.4103/2152-7806.170472)

7 Berwaerts J, Verhelst J, Mahler C \& Abs R. Cushing's syndrome in pregnancy treated by ketoconazole: case report and review of the literature. Gynecological Endocrinology 199913 175-182. (https://doi. org/10.3109/09513599909167552)

8 Boronat M, Marrero D, López-Plasencia Y, Barber M, Schamann Y \& Nóvoa FJ. Successful outcome of pregnancy in a patient with Cushing's disease under treatment with ketoconazole during the first trimester of gestation. Gynecological Endocrinology 201127 675-677. (https://doi.org/10.3109/09513590.2010.521268)

9 Connell JM, Cordiner J, Davies DL, Fraser R, Frier BM \& McPherson SG. Pregnancy complicated by Cushing's syndrome: potential hazard of metyrapone therapy. Case report. British Journal of Obstetrics and Gynaecology 198592 1192-1195. (https://doi. org/10.1111/j.1471-0528.1985.tb03037.x)

10 Costenaro F, Rodrigues TC, Lima PB de, Ruszczyk J, Rollin G \& Czepielewski MA. A successful case of Cushing's disease pregnancy treated with ketoconazole. Gynecological Endocrinology 201531 176-178. (https://doi.org/10.3109/09513590.2014.995615)

11 Jolly K, Darr A, Arlt W, Ahmed S \& Karavitaki N. Surgery for Cushing's disease in pregnancy: our experience and a literature review. Annals of the Royal College of Surgeons of England 2019101 e26-e31. (https://doi.org/10.1308/rcsann.2018.0175)

12 Leiba S, Weinstein R, Shindel B, Lapidot M, Stern E, Levavi H, Rusecki Y \& Abramovici A. The protracted effect of o, $\mathrm{p}^{\prime}$-DDD in Cushing's disease and its impact on adrenal morphogenesis of young human embryo. Annales d'Endocrinologie 198950 49-53.

13 Lim WH, Torpy DJ \& Jeffries WS. The medical management of Cushing's syndrome during pregnancy. European Journal of Obstetrics, Gynecology, and Reproductive Biology 2013168 1-6. (https://doi. org/10.1016/j.ejogrb.2012.12.015)

14 Giurgescu C. Are maternal cortisol levels related to preterm birth? Journal of Obstetric, Gynecologic, and Neonatal Nursing 200938 377-390. (https://doi.org/10.1111/j.1552-6909.2009.01034.x)

15 Mercer BM, Macpherson CA, Goldenberg RL, Goepfert AR, Hauguel-de Mouzon S, Varner MW, Iams JD, Meis PJ, Moawad AH, Miodovnik $\mathrm{M}$ et al. Are women with recurrent spontaneous preterm births different from those without such history? American Journal of Obstetrics and Gynecology 2006194 1176-1184; discussion 1184-1185. (https://doi.org/10.1016/j.ajog.2006.01.069)

16 Glynn LM, Schetter CD, Chicz-DeMet A, Hobel CJ \& Sandman CA. Ethnic differences in adrenocorticotropic hormone, cortisol and corticotropin-releasing hormone during pregnancy. Peptides 200728 1155-1161. (https://doi.org/10.1016/j.peptides.2007.04.005)

17 Bothou C, Anand G, Li D, Kienitz T, Seejore K, Simeoli C, Ebbehoj A, Ward EG, Paragliola RM, Ferrigno R et al. Current management and outcome of pregnancies in women with adrenal insufficiency: experience from a multicenter survey. Journal of Clinical Endocrinology and Metabolism 2020105 e2853-e2863. (https://doi.org/10.1210/ clinem/dgaa266)

18 Billionnet C, Mitanchez D, Weill A, Nizard J, Alla F, Hartemann A \& Jacqueminet $S$. Gestational diabetes and adverse perinatal outcomes from 716,152 births in France in 2012. Diabetologia 201760 636-644. (https://doi.org/10.1007/s00125-017-4206-6)

19 Blondel B, Coulm B, Bonnet C, Goffinet F, Le Ray C \& National Coordination Group of the National Perinatal Surveys. Trends in perinatal health in metropolitan France from 1995 to 2016: results from the French National Perinatal Surveys. Journal of Gynecology Obstetrics and Human Reproduction 201746 701-713. (https://doi. org/10.1016/j.jogoh.2017.09.002)

20 Castinetti F, Guignat L, Bouvattier C, Samara-Boustani D \& Reznik Y. Group 4: replacement therapy for adrenal insufficiency. Annales d'Endocrinologie 201778 525-534. (https://doi.org/10.1016/j. ando.2017.10.007)

21 Delabaere A, Huchon C, Deffieux X, Beucher G, Gallot V, Nedellec S, Vialard F, Carcopino X, Quibel T, Subtil D et al. Épidémiologie des pertes de grossesse [Epidemiology of loss pregnancy]. Journal de Gynécologie Obstétrique et Biologie de la Reproduction 201443 764-775. (https://doi.org/10.1016/j.jgyn.2014.09.011)

22 Crépin G \& Bréart G. Mortalité maternelle et mortalité périnatale des enfants nés à terme en France [Maternal and perinatal mortality of term deliveries in France]. Bulletin de l'Académie Nationale de Médecine 2010194 1581-1599. (https://doi.org/10.1016/S00014079(19)32186-7)

Received 23 October 2020

Revised version received 17 January 2021

Accepted 22 January 2021 